\title{
Causative agent distribution and antibiotic therapy assessment among adult patients with community acquired pneumonia in Chinese urban population
}

\author{
Youning Liu*1, Minjun Chen², Tiemei Zhao', Hui Wang2, Rui Wang3, \\ Baiqiang $\mathrm{Cai}^{4}$, Bin $\mathrm{CaO}^{4}$, Tieying Sun ${ }^{5}$, Yunjian $\mathrm{Hu}^{6}$, Qingyu Xiu ${ }^{7}$, Xin Zhou ${ }^{8}$, \\ Xing Ding ${ }^{8}$, Lan Yang ${ }^{9}$, Jiansheng Zhuo ${ }^{9}$, Yingchun Tang ${ }^{10}$, Kouxing Zhang ${ }^{10}$, \\ Derong Liang11, Xiaoju Lv'11, Shengqi Li ${ }^{12}$, Yong Liu ${ }^{13}$, Yunsong $\mathrm{Yu}^{14}$, \\ Zeqing Wei ${ }^{14}$, Kejing Ying ${ }^{15}$, Feng Zhao ${ }^{15}$, Ping Chen ${ }^{16}$ and Xiaona Hou ${ }^{17}$
}

\begin{abstract}
Address: ${ }^{1}$ Department of Respiratory Diseases, Chinese PLA General Hospital, Beijing, PR China, ${ }^{2}$ Department of Clinical Laboratory, Beijing Union Medical College Hospital, Beijing, PR China, ${ }^{3}$ Clinical Pharmacological Laboratory, Chinese PLA General Hospital, Beijing, PR China, ${ }^{4}$ Department of Respiratory Diseases, Beijing Union Medical College Hospital, Beijing, PR China, ${ }^{5}$ Department of Respiratory Diseases, Beijing Hospital, Beijing, PR China, ${ }^{6}$ Department of Clinical Laboratory, Beijing Hospital, Beijing, PR China, ${ }^{7}$ Department of Respiratory Diseases, Changzheng Hospital, Second Military Medical College, Shanghai, PR China, ${ }^{8}$ Department of Respiratory Diseases, First People's Hospital of Shanghai, Shanghai, PR China, ' Department of Respiratory Diseases, First Affiliated Hospital of Xi'an Jiaotong University, Xi'an, PR China, ${ }^{10}$ Department of Respiratory Diseases, Third Affiliated Hospital of Sun Yat-Sen University, Guangzhou, PR China, ${ }^{11}$ Clinical Pharmacological Institutes, West China Hospital of Sichuan University, Sichuan, PR China, ${ }^{12}$ Department of Respiratory Diseases, Second Affiliated University of China Medical University, Shenyang, PR China, ${ }^{13}$ Department of Clinical Laboratory, Second Affiliated University of China Medical University, Shenyang, PR China, ${ }^{14}$ Department of Infectious Diseases, First Affiliated Hospital of Zhejiang University, Zhejiang, PR China, ${ }^{15}$ Department of Respiratory Diseases, Sir Run Run Shaw Hospital, Zhejiang, PR China, ${ }^{16}$ Department of Respiratory Diseases, General Hospital of Shenyang Military Region, Shenyang, PR China and ${ }^{17}$ Department of Clinical Laboratory, General Hospital of Shenyang Military Region, Shenyang, PR China
\end{abstract}

Email: Youning Liu* - liuyn@301hospital.com.cn; Minjun Chen - chmj1933@hotmail.com; Tiemei Zhao - ztm1111@sina.com; Hui Wang - wh_bj@tom.com; Rui Wang - wangrui301@vip.sina.com; Baiqiang Cai - caibq@hotmail.com; Bin Cao - caobin1999@gmail.com; Tieying Sun - suntieying3@hotmail.com; Yunjian Hu - huyunjian@sina.com; Qingyu Xiu - xiu_qingyu@126.com; Xin Zhou -xzhou53@163.com; Xing Ding - xzhou53@163.com; Lan Yang - ylan8@medmail.com.cn;

Jiansheng Zhuo - ylan8@medmail.com.cn; Yingchun Tang - tangyingchun@163.net; Kouxing Zhang - kxz6210@tom.com; Derong Liang - miaosiyi@yahoo.com.cn; Xiaoju Lv - lvxj3396@yahoo.com.cn; Shengqi Li - Lxn_19999@sohu.com;

Yong Liu - Lxn_19999@sohu.com; Yunsong Yu - zeqingw@163.com; Zeqing Wei - zeqingw@163.com; Kejing Ying - yingsrrsh@163.com; Feng Zhao - yingsrrsh@163.com; Ping Chen - hxnk2004@126.com; Xiaona Hou - xiaonahou@sohu.com

* Corresponding author

Published: 18 March 2009

BMC Infectious Diseases 2009, 9:31 doi:10.1/86/|47|-2334-9-31
Received: 12 October 2008

Accepted: 18 March 2009

This article is available from: http://www.biomedcentral.com/147I-2334/9/3I

(C) 2009 Liu et al; licensee BioMed Central Ltd.

This is an Open Access article distributed under the terms of the Creative Commons Attribution License (http://creativecommons.org/licenses/by/2.0), which permits unrestricted use, distribution, and reproduction in any medium, provided the original work is properly cited.

\begin{abstract}
Background: Knowledge of predominant microbial patterns in community-acquired pneumonia (CAP) constitutes the basis for initial decisions about empirical antimicrobial treatment, so a prospective study was performed during 2003-2004 among CAP of adult Chinese urban populations.
\end{abstract}

Methods: Qualified patients were enrolled and screened for bacterial, atypical, and viral pathogens by sputum and/or blood culturing, and by antibody seroconversion test. Antibiotic treatment and patient outcome were also assessed. 
Results: Non-viral pathogens were found in 324/610 (53.1\%) patients among whom M. pneumoniae was the most prevalent (126/610, 20.7\%). Atypical pathogens were identified in 62/195 (31.8\%) patients carrying bacterial pathogens. Respiratory viruses were identified in 35 (19\%) of 184 randomly selected patients with adenovirus being the most common (16/184, 8.7\%). The nonsusceptibility of S. pneumoniae to penicillin and azithromycin was $22.2 \%$ (Resistance (R): $3.2 \%$, Intermediate (I): $19.0 \%$ ) and $79.4 \%$ (R: $79.4 \%$, I: $0 \%$ ), respectively. Of patients ( $3 \mathrm{I} 2$ ) from whom causative pathogens were identified and antibiotic treatments were recorded, clinical cure rate with $\beta$-lactam antibiotics alone and with combination of a $\beta$-lactam plus a macrolide or with fluoroquinolones was $63.7 \%$ (79/I24) and 67\%(I26//88), respectively. For patients having mixed $M$. pneumoniae and/or $C$. pneumoniae infections, a better cure rate was observed with regimens that are active against atypical pathogens (e.g. a $\beta$-lactam plus a macrolide, or a fluoroquinolone) than with $\beta$-lactam alone (75.8\% vs. $42.9 \%, p=0.045)$.

Conclusion: In Chinese adult CAP patients, $M$. pneumoniae was the most prevalent with mixed infections containing atypical pathogens being frequently observed. With $S$. pneumoniae, the prevalence of macrolide resistance was high and penicillin resistance low compared with data reported in other regions.

\section{Background}

Community-acquired pneumonia (CAP) remains a common disease with high mobidity, mortality, and treatment cost $[1,2]$. The microbial patterns reported for CAP differ considerably, depending on epidemiologic area, patient populations, and the extent and nature of the microbiologic techniques used. Knowledge of predominant microbial patterns in CAP constitutes the basis for initial decisions about empirical antimicrobial treatment [3]. However, no prospective study has been done to investigate such patterns in a large Chinese population over several districts at the same time.

In the present work, we conducted a prospective study of the etiology of CAP in adult patients at 12 centers in 7 Chinese cities to assess the causative microbial spectrum, the influence of patient age, previous antibiotic use, and the Pneumonia Patient Outcomes Research Team (PORT) category of pneumonia on microbial patterns in the disease [4]. Antimicrobial susceptibility of isolates from clinical specimens was also tested to estimate the prevalence of drug resistance of common CAP-causing bacteria, especially Streptococcus pneumoniae and Haemophilus influenzae. Meanwhile the outcome of antibiotic therapy for CAP was analyzed. The results revealed a different predominant microbial spectrum and relatively higher penicillin susceptibility of $S$. pneumoniae in the Chinese population than previously reported $[5,6]$.

\section{Methods}

\section{General information}

A prospective study of the causes and antimicrobial treatment outcome of CAP was conducted in consecutively enrolled adult patients (age $\geq 18$ years) between 1 December 2003 and 30 November 2004 at 12 centers in 7 Chinese cities (Beijing, Shanghai, Shenyang, Xi'an, Chengdu,
Guangzhou, and Hangzhou). The cities were selected according to their geographical locations: Beijing and Shenyang lie in the north area, Guangzhou in the south area, Shanghai and Hangzhou in the east area, and $\mathrm{Xi}^{\prime}$ an and Chengdu in the west area. The study year was divided into four periods: December to February, March to May, June to August, and September to November. Two central laboratories, PLA General Hospital laboratory and Beijing Union Medical College Hospital laboratory, conducted pathogen identification and drug susceptibility testing. Therapy was recommended according to the CAP guidelines from the Chinese Medical Association [7], but the final drug choice was determined by attending physicians. Informed consent was obtained from each patient before sputum and blood sample collection. The protocol was approved by the Ethics Committee for Clinical Studies at PLA General Hospital (Protocol: 2003-\#035).

\section{Diagnostic criteria}

According to the guidelines from the Chinese Medical Association [7], CAP was defined as an acute infiltrate on a chest radiograph and at least one of the following: (a) new or escalating cough, sputum production, purulent sputum, chest pain; (b) fever; (c) altered breath sounds or/and rales; (d) white blood cell count: $>10 \times 10^{9} / \mathrm{L}$ or $<$ $4 \times 10^{\%} / \mathrm{L}$. Patients were excluded if they had tuberculosis, lung cancer, non-infective interstitial lung diseases, pulmonary oedema, pulmonary lobe collapse, pulmonary thromboembolism, pulmonary infiltration with eosinophilia, or pulmonary angiitis. Atypical pathogens defined as Mycoplasma pneumoniae, Chlamydophila pneumoniae, and Legionella spp. The microbial etiology of pneumonia was considered if one of the following criteria was met: (1) valid sputum sample generating one or more predominant microbial pathogens; (2) blood culture producing a bacterial pathogen; (3) serologic conversion: e.g. a $\geq$ 
4-fold increase in titers of antibodies to Mycoplasma pneumoniae, Chlamydophila pneumoniae, Legionella pneumophila, or respiratory viruses (influenza virus A and B, adenovirus, and respiratory syncytial virus).

\section{Microbiological evaluation}

Regular sputum sampling was done with most (590/610) patients enrolled in the study, and blood samples were taken if patients had a fever of $>38.5^{\circ} \mathrm{C}$. Sputum was Gram-stained. Representative sputum originated from the lower respiratory tract was defined as that containing $>25$ granulocytes and $<10$ epithelial cells per low power field microscopic view. Validated sputum and blood samples were cultured; microorganisms were identified according to standard methods [8].

Blood samples $(6-8 \mathrm{ml})$ were collected aseptically from patients. Acute sera were separated and stored at $-80^{\circ} \mathrm{C}$ until convalescent sera, obtained 2 to 4 weeks after the acute specimen, were available for simultaneous testing [9]. The antibody titer to M. pneumoniae was determined by microparticle agglutination using the Serodia Myсо $^{\circledR}$ commercial kit (Fujirebio, Japan). C. pneumoniae-specific IgG antibody titers were determined by the indirect immunofluorescence method using the FI2192-1010G commercial kit (Euroimmun, Germany), as were antibodies to 14 different serogroups of L. pneumophila. Respiratory virus antibodies (IgG) were detected by ELISA using the Virion-Serion commercial kit (Febricant, Germany).

\section{Antimicrobial susceptibility testing}

MICs of isolates were determined at Beijing Union Medical College Hospital laboratory, using agar dilution according to CLSI protocols. Breakpoint concentrations used to interpret MIC data qualitatively were based upon those published by the National Committee for Clinical Laboratory Standards of the USA (NCCLS, 2004) [10]. The inocula comprised $10^{4}-10^{5} \mathrm{cfu} / \mathrm{spot}$, delivered with a multipoint inoculator. Cultures were incubated with antimicrobial agents selected on the basis of common empiric therapy options in China.

\section{Data collection}

Clinical, radiographic, and laboratory data were recorded on a data sheet and entered into a computer database. The following data were recorded on enrolling: age, gender, comorbidities, antimicrobial treatment prior to enrolment, duration of symptoms before the diagnosis of pneumonia, clinical symptoms (body temperature, pleuritic chest pain, purulent sputum), vital signs (respiratory rate, heart rate, arterial systolic and diastolic blood pressure, presence of rales), haematology (total WBC with differential counts, platelet count, hemoglobin), chest radiographic pattern, and alcohol consumption.
Data on modifying or risk factors collected, included underlying cardiopulmonary diseases, exposure to a child in a day care center, residence in a nursing home, $\beta$-lactam therapy within the past 3 months, broad-spectrum antibiotic therapy for $>7 \mathrm{~d}$ in the past two months, corticosteroid therapy (> $10 \mathrm{mg}$ of prednisone per day), alcoholism, previous pneumonia history, and malnutrition.

\section{Antibiotic therapy analysis}

According to the causative pathogens identified, we classified the patients into four groups for analysis of outcome of antibiotic therapy. First group: M. pneumoniae and $C$. pneumoniae mono- and co-infection; second group: bacterial co-infection with $M$. pneumoniae, C. pneumoniae or both; third group: L. pneumophila mono-infection or mixed with other pathogens; and fourth group: bacterial infection only. Clinical response, evaluated post-treatment, was rated as cure (complete resolution of all signs and symptoms of pneumonia), improvement, or lack of progression for all abnormalities on chest radiograph [11].

\section{Statistical analysis}

Statistical analysis was carried out using SAS (SAS Institute, Cary, NC, USA) version 8.2. Results are presented descriptively (some expressed as mean $\pm \mathrm{SD}$ ). Data were compared by use of the $\chi^{2}$ statistic or Fisher's exact test. For all analyses, $p<0.05$ was considered to be significant.

\section{Results}

\section{General information}

The 610 enrolled patients (382 male and 228 female) had a mean age of $52.4( \pm 20.0 \mathrm{SD})$ years. When stratified into the five classes of the PORT cumulative point system [4], most patients were in low-risk Classes I (33.8\%), II (36.2\%) and III (15.9\%); moderate-risk Class IV disease was determined in $13.1 \%$ of patients, and high-risk Class $\mathrm{V}$ in only $1.0 \%$. Clinical symptoms, as well as modifying and risk factors, are summarized in Table 1 . Three hundred forty two patients (56.1\%) received antimicrobial treatment prior to enrollment. Thirty eight percent of patients had at least one co-morbid illness, with cardiac co-morbidity being the most frequent condition $(25.9 \%)$, followed by pulmonary co-morbidity (12.8\%).

\section{Pathogens distribution}

Of 610 patients, sputum samples were obtained in 590 patients, and blood samples for culture were available in 141 patients. Of the 590 patients with sputum samples, $S$. pneumoniae was the most common bacterium identified, accounting for $10.3 \%$, followed by $H$. influenzae $(9.2 \%)$.

Overall, non-viral pathogens were detected in 324 of the 610 patients (Table 2). Of these, 195 were detected by sputum examination, 5 by hemoculture, and 191 by serol- 
Table I: Summary of characteristics of all enrolled patients with community-acquired pneumonia $(n=610)$

\begin{tabular}{lc}
\hline & $n(\%)^{*}$ \\
\hline Symptoms & $455(74.6)$ \\
Fever & $582(95.4)$ \\
Cough & $536(87.9)$ \\
Purulent sputum & $402(65.9)$ \\
Rales & $260(42.6)$ \\
Leukocytosis $>10 \times 109 / L$ & $7(1.2)$ \\
Modifying factors & $120(19.7)$ \\
Exposure to a child in a day care center & $61(10.0)$ \\
B-lactam therapy within the past 3 months & $9(1.5)$ \\
Broad-spectrum antibiotic therapy for $>7$ d in the past two months & $1(0.2)$ \\
Corticosteroid therapy $(>10$ mg of prednisone per day) & $79(13.0)$ \\
Alcoholism & $158(25.9)$ \\
History of pneumonia & $78(12.8)$ \\
Comorbidities & $33(5.4)$ \\
Cardiac & $25(4.1)$ \\
Pulmonary & $8(1.3)$ \\
Diabetes mellitus & $7(1.1)$ \\
Central nervous system & \\
Renal & \\
Hepatic & \\
\hline
\end{tabular}

*Patient number, numbers in parenthesis are percentage of total patient number.

ogy testing. The most common agent identified was $M$. pneumoniae, which was found in 126 patients $(20.7 \%) . S$. pneumoniae was identified in 63 patients $(10.3 \%)$, followed by $H$. influenzae in 56 patients $(9.2 \%)$. The frequency of other infective agents was $6.6 \%$ for $C$. pneumoniae (40 patients), 6.1\% for Klebsiella pneumoniae (37 patients), 5.1\% for L. pneumophila (31 patients), 3.8\% for Staphylococcus aureus (23 patients), 1.6\% for Escherichia coli (10 patients), $1.3 \%$ for Moraxella catarrhalis (8 patients), and $1.0 \%$ for Pseudomonas aeruginosa (6 patients). Atypical pathogens accounted for 31.3\% overall. In 184 patients whose sera antibody response to respiratory viruses were analyzed, 35 patients (19.0\%) were tested positive for at least one viral infection. The distribution of viruses was listed in Table 3. Overall, adenovirus was the most common, identified in 16 patients $(8.7 \%)$, followed by influenza virus B in 12 patients (6.5\%), respiratory syncytial virus in 8 patients $(4.3 \%)$, and influenza virus $A$ in 5 patients $(2.7 \%)$. In patients that tested positive for viral infections, mixed infections with an atypical

Table 2: Non-viral pathogens detected among 610 patients with community-acquired pneumonia

\begin{tabular}{lc}
\hline Pathogen & Patients, $\mathrm{n}(\%)$ \\
\hline Mono-infection & $82(13.4)$ \\
Mycoplasma pneumoniae & $37(6.07)$ \\
Streptococcus pneumoniae & $33(5.4)$ \\
Haemophilus influenzae & $29(4.8)$ \\
Chlamydophila pneumoniae & $23(3.8)$ \\
Klebsiella pneumoniae & $17(2.8)$ \\
Staphylococcus aureus & $17(2.8)$ \\
Legionella pneumophila & $6(1.0)$ \\
Escherichia coli & $5(0.8)$ \\
Pseudomonas aeruginosa & $5(0.8)$ \\
Moraxella catarrhalis & $70(11.5)$ \\
Mixed infections & $64(10.5)$ \\
$\quad$ Two pathogens & $5(0.8)$ \\
Three pathogens & $1(0.2)$ \\
Four pathogens: & $324(53.1)$
\end{tabular}

Note: Percentage of the most three common agents in which pathogens were identified was $38.9 \%$ for M. pneumoniae, $19.4 \%$ for S. pneumoniae, and $17.3 \%$ for $H$. influenzae. 
Table 3: Respiratory viruses detected among I 84 patients with community-acquired pneumonia

\begin{tabular}{ll}
\hline Pathogen & Patients, $\mathrm{n}(\%)$ \\
\hline $\begin{array}{l}\text { Mono-virus infection } \\
\text { influenza virus B }\end{array}$ & $6(3.3)$ \\
$\quad$ adenovirus & $3(1.6)$ \\
$\quad$ respiratory syncytial virus & $2(1.1)$ \\
$\quad$ influenza virus+ adenovirus & $1(0.5)$ \\
Mixed infections & $15(8.2)$ \\
$\quad$ Two pathogens & $6(3.3)$ \\
Three pathogens & $2(1.1)$ \\
Four pathogens & $35(19.0)$ \\
Total & \\
\hline
\end{tabular}

Note: Percentage of adenovirus in which viruses were identified was $45.7 \%$, and $34.3 \%$ for influenza virus B, $22.9 \%$ for respiratory syncytial virus, $14.3 \%$ for influenza virus $A$.

pathogen or a bacterium were detected in 23 patients $(65.7 \%)$, accounting for $12.5 \%$ of the total 184 patients tested for viral infection. The most common pathogen involved in co-infection with virus was M. pneumonia, accounting for 8 patients. The most common bacterium found in co-infection was $H$. influenzae, accounting for 4 patients.

In addition to co-infection with respiratory viruses, mixed infections were present in 70 of the 610 patients whose microbial etiology was determined. Among these 70 patients, co-infection with two, three, and four pathogens was detected in $64(91.4 \%), 5(7.1 \%)$, and $1(1.4 \%)$ patients, respectively. Among the 63 patients from whom $S$. pneumoniae was identified, co-infection was detected in $26(41.3 \%)$ patients (i.e.: M. pneumoniae in 15 (23.8\%), C. pneumoniae in $3(4.8 \%)$, and other pathogens in 8 $(12.7 \%))$. Where M. pneumoniae infection was detected, a co-pathogen was diagnosed in 44 (34.9\%) cases (S. pneumoniae in 15, $H$. influenzae in 13, and other pathogens in 16). Among the 195 patients with bacterial infection, an atypical co-pathogen was identified in $62(31.8 \%)$ cases.

M. pneumoniae were found less often in the elderly than in patients aged $\leq 50$ years $(13.4 \%$ vs. $30.0 \%, p<0.05)$. Comorbidity was found in $27.8 \%$ of M. pneumoniae-infected patients. Age or co-morbidity alone had no impact on $C$. pneumoniae infection, but $C$. pneumoniae was more frequently found in elderly patients ( $>70$ years) without comorbid illness than in those with co-morbidity $(18.4 \%$ $(7 / 38)$ vs. $6.3 \%(7 / 112), p<0.05)$. Respiratory virus infection was less frequent in patients aged $>70$ years than in patients $\leq 70$ years old ( $0 \%$ vs. $24.6 \%, p<0.05)$. In patients aged $>50$ years, $K$. pneumoniae infection was more frequent than in those aged $\leq 50$ years $(8.5 \%$ vs. $3.0 \%, p<0.05$ ). Five of the 6 patients with $P$. aeruginosa infection were aged $>70$ years. In patients with cardiovascular or pulmonary co-morbidity, $12.4 \%(21 / 170)$ had $K$. pneumoniae or $P$. aeruginosa infections, compared to only $5.0 \%(22 / 440)$ of patients without these co-morbidities ( $p$ $<0.05)$.

$56.1 \%$ of patients received antibiotics before enrolling. $H$. influenzae was 6-fold more frequently isolated from patients who had not received antibiotics before enrolling than those who had been treated with antibiotics $(17.2 \%$ $(46 / 268)$ vs. $2.9 \%(10 / 342), p<0.05)$. Prior antibiotic therapy did not significantly reduce the frequency of $S$. pneumoniae isolation.

Patients of PORT Classes I and II were more likely to have $S$. pneumoniae and $H$. influenzae etiology. In patients with Class V disease, association with $K$. pneumoniae was evident. M. pneumoniae was common in Class I, II, and III disease, especially in Class I. With regard to seasonal variations, isolation rate of common pathogens did not show any statistically significant differences in the four period of our survey.

\section{Antimicrobial susceptibility}

A total of 63 isolates of $S$. pneumoniae were submitted for antimicrobial susceptibility testing. The proportions of intermediately or fully resistant isolates to penicillin were $19.0 \%$ and $3.2 \%$, respectively. The prevalence of isolates considered non-susceptible to azithromycin and new fluroquinolones were $79.4 \%$ and $\leq 6.3 \%$, respectively (Table 4). In 56 isolates of $H$. influenzae tested, $8.4 \%$ produced beta-lactamase and $11.9 \%$ were non-susceptible to ampicillin.

Table 4: Antimicrobial susceptibility of $63 \mathrm{~S}$. pneumoniae isolates obtained in the study

\begin{tabular}{|c|c|c|c|c|c|c|}
\hline \multirow[t]{2}{*}{ Antimicrobial agent } & \multicolumn{3}{|c|}{$\%$ of isolates } & \multicolumn{3}{|c|}{ MIC $(\mu \mathrm{g} / \mathrm{ml})$} \\
\hline & Susceptible & Intermediate & Resistant & $\mathrm{MIC}_{50}$ & $\mathrm{MIC}_{90}$ & Range \\
\hline Penicillin & 77.8 & 19.0 & 3.2 & 0.016 & 0.5 & $0.016-4$ \\
\hline Azithromycin & 20.6 & 0.0 & 79.4 & 256 & $>256$ & $0.032-512$ \\
\hline Amoxycillin/clavulanic acid & 95.2 & 3.2 & 1.6 & 0.032 & I & $0.032-8$ \\
\hline Levofloxacin & 93.7 & 0.0 & 6.3 & I & 2 & $0.5-16$ \\
\hline Gatifloxacin & 93.7 & 0.0 & 6.3 & 0.25 & 0.5 & $0.125-4$ \\
\hline Moxifloxacin & 95.2 & 3.2 & 1.6 & 0.125 & 0.25 & $0.064-4$ \\
\hline
\end{tabular}




\section{Antibiotics usage and clinical outcome}

In our study, the most frequently administered antibiotics were $\beta$-lactams, accounting for $73.6 \%$ (449/610), followed by fluoroquinolones $(49.8 \%, 304 / 610)$ and macrolides $(22.8 \%, 139 / 610)$. There was a total of 315 patients whose infecting pathogen was identified and antibiotic usage was recorded. Since only 3 patients used a macrolide as monotherapy, the clinical outcome of these patients was not considered. In the other 312 patients, the clinical cure rate with $\beta$-lactam antibiotics was $63.7 \%(79 / 124)$, with combination of a $\beta$-lactam plus a macrolide or with fluoroquinolone (either alone or in combination with other antibiotics) was 67\% (126/188). For patients affected by atypical pathogens, cure rates are presented in Table 5. For mixed infections involving bacteria plus M. pneumoniae and/or C. pneumoniae, $\beta$-lactams plus a macrolide or fluoroquinolone (alone or combined with other antibiotics, e.g. regimens that are active against atypical pathogens) showed a statistically significant higher cure rate than $\beta$-lactams alone $(75.8 \%$ v.s. $42.9 \%$, $p=0.045)$. In patients with co-morbid illness, the total cure rate of therapy with the combination of a $\beta$-lactam plus a macrolide or with fluoroquinolone was not significantly greater than that with $\beta$-lactams (40.4\% vs. $50.0 \%$, $p>0.05)$.

\section{Discussion}

The distribution of pathogens associated with CAP is attracting world-wide attention. The present study is the first in China to prospectively investigate the pathogen distribution in a large, broadly distributed patient population. The study comprised several representative districts and covered all seasons of the year. The data reveal a unique pattern of pathogen distribution in CAP patients in China and show a relatively higher susceptibility of $S$. pneumoniae isolates to penicillin but relatively lower susceptibility to fluoroquinolones than that reported in Europe and North America.
In previous studies, 30 to $60 \%$ of the CAP cases did not yield an identifiable pathogen [12], in line with our results. Although $S$. pneumoniae remains the most prevalent or frequently isolated etiologic agent in cases of CAP $[2,13]$, other organisms, such as $H$. influenzae and $M$. catarrhalis, as well as the so-called atypical pathogens, including M. pneumoniae, C. pneumoniae, and L. pneumophila, are now being reported more frequently than in the past $[14,15]$. Atypical pathogens are responsible for 30 to $40 \%$ of cases of CAP [16]. In some reports, the infection with atypical pathogens was more than with $S$. pneumoniae, especially for M. pneumoniae. Moola et al [15] reported that when pathogens were confirmed in 131 of 504 patients, M. pneumoniae was found in 25\% patients, and $S$. pneumoniae in $22 \%$. In a study performed in Spain [16], M. pneumoniae was the most frequent pathogen (33\%), with S. pneumoniae being found in only 19 of 110 patients with CAP.

In our study, the rate of atypical pathogen infection is $31.3 \%$ (if convalescent sera obtained longer than 4 weeks after the acute specimen, L. pneumophila may be more, and this percentage may be higher); M. pneumoniae is the most prevalent etiologic agent, accounting for $20.7 \%$ of CAP cases, i.e. more than those associated with $S$. pneumoniae. Such finding is similar to our previous study between 2001 and 2002 in Beijing [9,17]. Previous reports show that viral infection in patients with CAP varies from $4 \%$ to $39 \%$ [18], the most frequent being influenza spp. In the present study, respiratory viruses account for $19.0 \%$ of tested patients, with adenovirus being the most common.

Previous studies have shown that some patients with CAP can have mixed infections involving both bacterial and atypical pathogens [2,19]. C. pneumoniae is frequently found in mixed infections and has been detected in over half of cases with $S$. pneumoniae in reports from other countries $[2,19]$. In our study, however, M. pneumoniae is the most frequent co-infecting pathogen. Multiple simul-

Table 5: Cure rate [n (\%)] of antibiotics in patients with CAP affected by atypical pathogens

\begin{tabular}{ccc}
\hline Pathogens & $\beta$-lactam alone & $\begin{array}{c}\beta \text {-lactam plus macrolides } \\
\text { or fluoroquinolone (alone } \\
\text { or combine with other } \\
\text { antibiotics) }\end{array}$ \\
\hline$M P, C P, M P+C P$ & $25(62.5)$ & $45(73.8)$ \\
\hline $\begin{array}{c}\text { MP+bacteria, } \\
\text { MP+bacteria, }\end{array}$ & $6(42.9)$ & $25\left(75.8^{*}\right)$ \\
\hline LP or LP mixed with other pathogens & $8(61.5)$ & $10(55.6)$
\end{tabular}

MP: Mycoplasma pneumoniae; CP: Chlamydophila pneumoniae; LP: Legionella pneumophila

*compared to $\beta$-lactam alone, $p=0.045$ 
taneous infections might interfere with the pulmonary cleansing function and thus help establish setting for CAP [20]. Pathogens such as C. pneumoniae induce ciliostasis in human bronchial epithelial cells [21], and M. pneumoniae exerts a toxic effect on ciliated human epithelium [22]. Currently, the clinical implications of mixed infections are still undetermined. Influenza and other viruses can cause primary viral pneumonia; secondary bacterial infections are common in hospitalized adults, and the reported frequency has ranged widely, from $26 \%$ to $77 \%$ in different studies [18]. The common cause of bacterial superinfection is $S$. pneumoniae. In our study, mixed infection of respiratory viruses with other pathogens accounts for $12.5 \%$. The most common co-infecting pathogen was $M$. pneumoniae.

The impact of age is restricted to an association of younger patients (age $\leq 50$ years) with $M$. pneumoniae. This finding is consistent with several previous studies $[23,24]$, which show that $M$. pneumoniae is the most common etiologic agent in the 17-44 year age group. Patients without comorbid illnesses are also more likely to have M. pneumoniae infection. In our study, only $27.8 \%$ patients of $M$. pneumoniae have co-morbid illnesses. It has been reported that $C$. pneumoniae infection is more likely to occur in elderly persons with co-morbid disease than in those who are otherwise healthy [25]. However, our data showed a higher incidence of $C$. pneumoniae infection in older patients without co-morbid illness than with, contradicting previous conclusion. An association of older age with Gram-negative pathogens has been observed by several groups $[12,26]$. It is well known that colonization of the oropharyngeal mucosa by aerobic Gram-negative bacilli increases with age [27]. In our study an age of $>50$ years was significantly associated with these pathogens. Pulmonary co-morbidity also predisposes to pneumonia due to $K$. pneumoniae or $P$. aeruginosa, probably due to previous tracheabronchial colonization in COPD [28]. In our study, Staphylococcus aureus was more frequent in PORT I than in PORTs II through IV, probably due to patients having low co-morbidities of diabetes mellitus and to sample size being too small.

Antibiotic resistance in pneumococci is now considered to be a global problem. The ANSORP (Asian network for surveillance of resistant pathogens) study, conducted in Asian countries from September 1996 to June 1997, showed that the non-suscepibility to penicillin was as high as $79.7 \%$ in Korea and non-susceptibility to erythromycin was as high as $90.5 \%$ in Taiwan [29]. In China, penicillin-non-susceptibile $S$. pneumoniae ranged from $13.9 \%$ to $42.7 \%$, and erythromycin-non-susceptibile $S$. pneumoniae ranged from $42.5 \%$ to $83.6 \%$ in surveys conducted from 1997 to 2003 [5,6,14,30-32]. Among the 63 strains of $S$. pneumoniae obtained from the present study, non-susceptibility to penicillin was $22.2 \%$; non-susceptibility to azithromycin was 79.4\%. In 2008 year, CLSI changed the break point of penicillin for patients with non-CNS infections, according to this, the non-susceptibility to penicillin was only $3.2 \%$. Although new fluoroquinolones have good activity against $S$. pneumoniae, the resistance rate of new fluoroquinolones is higher in China than that reported in other countries except for Hong Kong [33], which is possibly a result of selective pressure due to increased quinolone use in China.

American Thoracic Society guidelines [34] advocate that all populations with CAP should be treated for possible infection with atypical pathogens. Macrolides are recommended as the first-line choice for outpatients. However, such a recommendation may not be suitable for patients in China because the prevalence of macrolide resistance is so high (about 70\% among S. pneumoniae isolates) and because ermB-mediated high-level resistance is more frequent than low-level resistance conferred by mefA $(79.1 \%$ contained the erm $\mathrm{B}$ gene, $10.8 \%$ contained the mefA gene, and $10.1 \%$ harbored both the $\operatorname{erm} \mathrm{B}$ and $m e f \mathrm{~A}$ genes) as shown in our previous study [6]. In the present study, for patients infected with M. pneumoniae and/or C. pneumoniae, the cure rate with $\beta$-lactams plus macrolides or with fluoroquinolones was not significantly better than with $\beta$ lactams alone. A meta-analysis also showed no advantage of antibiotics active against atypical pathogens over $\beta$ lactams [35]. Thus, $\beta$-lactams (penicillin, amoxicillin, cephalosporins) should remain agents of choice in the initial management of mild CAP without co-morbidity in China. But for moderate or severe infection or in patients with co-morbidities, therapy should be recommended with either a $\beta$-lactam/macrolide combination or an antipneumococcal fluoroquinolone alone [36-39].

\section{Conclusion}

In Chinese adult CAP patients, M. pneumoniae was the most prevalent with mixed infections containing atypical pathogens being frequently observed. With S. pneumoniae, the prevalence of macrolide resistance was high and penicillin resistance low compared with data reported in other regions. $\beta$-lactams (penicillin, amoxicillin, cephalosporins) should remain agents of choice in the initial management of mild CAP without co-morbidity in China.

\section{Competing interests}

The authors declare that they have no competing interests.

\section{Authors' contributions}

YL, TZ, BC, BC, TS, QX, XZ, LY, YT, DL, SL, YY, KY, and PC conceived the study, enrolled patients and collected data. MC, HW, RW, YH, XD, JZ, KZ, XL, YL, ZW, FZ, and XH conducted microorganisms identification, and $\mathrm{HW}$ 
responsible for antimicrobial susceptibility testing. All authors read and approved the final manuscript.

\section{Acknowledgements}

We would like to thank Xilin Zhao for critical comments on the manuscript. The work was supported by an unrestricted educational grant from the Bayer Pharmaceutical Company.

\section{References}

I. Hirani NA, Macfarlane JT: Impact of management guidelines on the outcome of severe community acquired pneumonia. Thorax 1997, 52:17-21.

2. Lim WS, Macfarlane JT, Boswell TCJ, Harrison TG, Rose D, Leinonen $M$, Saikku P: Study of community-acquired pneumonia aetiology (SCAPA) in adults admitted to hospital: implications for management guidelines. Thorax 200I, 56:296-30I.

3. Woodhead M, Blasi F, Ewig S, Huchon G, leven M, Ortqvist A, Schaberg T, Torres A, Heijden G van der, Verheij T): European Respiratory Society; European Society of Clinical Microbiology and Infectious Diseases. Guidelines for the management of adult lower respiratory tract infections. Eur Respir J 2005, 26:1138-1180.

4. Bartlett JG, Breiman RF, Mandell LA, File TM Jr: Communityacquired pneumonia in adults:guidelines for management. The Infectious Diseases Society of America. Clin Infect Dis 1998, 26:8II-838.

5. Shen X, Lu Q, Ye Q, Zhang G, Yu S, Zhang H, Deng Q, Yang Y: Prevalence of antimicrobial resistance of Streptococcus pneumoniae in Chinese children: four hospitals surveillance. Chin Med J (Engl) 2003, I | 6: | 304- I 307.

6. Tiemei Z, Xiangqun F, Youning L: Resistance phenotypes and genotypes of erythromycin-resistant Streptococcus pneumoniae isolates in Beijing and Shenyang, China. Antimicrob Agents Chemother 2004, 48:4040-404I.

7. Chinese Medical Association Respiratory Branch: Guidelines for diagnosis and treatment of connumity acquired pneumonia. Chin J Tuberc Respir Dis 1999, 22:199-201.

8. Murray PR, Baron EJ, Jorgensen JH, Pfaller MA, Yolken RH: Manual of Clinical Microbiology 8th edition. American Society for Microbiology Press; 2003.

9. Ngeow YF, Suwanjutha S, Chantarojanasriri T, Wang F, Saniel M, Alejandria M, Hsueh PR, Ping-Ing L, Park SC, Sohn JW, Aziah AM, Liu Y, Seto WH, Ngan CC, Hadiarto M, Hood A, Cheong YM: An Asian study on the prevalence of atypical respiratory pathogens in community-acquired pneumonia. Intern J Infect Dis 2005, 9:144-153.

10. National Committee for Clinical Laboratory Standards: Performance Standards for Antimicrobial Susceptibility Testing: Fourteenth Informational Supplement MI00-I 4. Pennsylvania 2004.

II. Brande P Van den, Vondra V, Vogel F, Schlaeffer F, Staley H, Holmes $C$ : Sequential therapy with cefuroxime followed by cefuroxime axetil in community-acquired pneumonia. Chest 1997, I I 2:406-4 I5.

12. Niederman MS, Bass JB Jr: Guidelines for the initial management of adults with community-acquired pneumonia: diagnosis, assessment of severity, and initial antimicrobial therapy: American Thoracic Society: Medical Section of the American Lung Association. Am Rev Respir Dis 1993, | 48: |4|8-| 426.

13. Gutierrez F, Masia M, Rodriguez JC, Ayelo A, Soldán B, Cebrián L, Mirete $C$, Royo G, Hidalgo AM: Evaluation of the immunochromatographic Binax NOW assay for detection of Streptococcus pneumoniae urinary antigen in a prospective study of community-acquired pneumonia in Spain. Clin Infect Dis 2003, 36:286-292.

14. Huang HH, Zhang YY, Xiu QY, Zhou X, Huang SG, Lu Q, Wang DM, Wang F: Community-acquired pneumonia in Shanghai, China: microbial etiology and implications for empirical therapy in a prospective study of $\mathbf{3 8 9}$ patients. Eur J Clin Microbiol Infect Dis 2006, 25:369-374.

15. Moola S, Hagberg L, Churchyard GA, Dylewski JS, Sedani S, Staley H: A multicenter study of grepafloxacin and clarithromycin in the treatment of patients with community-acquired pneumonia. Ches 1999, II 6:974-983.
16. Antela A, Guerrero A, Meseguer M, González-Sainz J, Escudero R, Pérez-Elías MJ, Quereda C, Hermida JM, Cobo J, Montilla P: Community-acquired pneumonia: prospective study of $10 \mathrm{I}$ adult, immunocompetent patients for I year. Enferm Infect Microbiol Clin 1993, II:525-530.

17. Liu YN, Zhao TM, Yao WZ, Zhang LS, He ZY, Jiao YM, Duan YY, Nie ZS, Wang R, Liu QF: Prevalence of atypical pathogens in adult patients with community-acquired pneumonia in Beijing. Chin J Tuberc Respir Dis 2004, 27:27-30.

18. Greenberg SB: Viral pneumonia. Infect Dis Clin North Am 199I, 5:603-62I.

19. Kauppinen MT, Herva E, Kujala $P$, Leinonen $M$, Saikku $P$, Syriälä $H$ : The etiology of community-acquired pneumonia among hospitalized patients during a Chlamydia pneumoniae epidemic in Finland. J Infect Dis 1995, I 72:1330-1335.

20. Lepow ML, Balassanian N, Emmerich J, Roberts RB, Rosenthal MS, Wolinsky E: Interrelationships of viral, mycoplasmal and bacterial agents in uncomplicated pneumonia. Am Rev Respir Dis 1968, 97:533-545.

21. Shemer-Avni Y, Lieberman D: Chlamydia pneumoniae-induced ciliostasis in ciliated bronchial epithelial calls. J Infect Dis 1995, I7I: 1274-I298

22. Collier AM, Clyde WA: Relationships between Mycoplasma pneumoniae and human respiratory epithelium. Infect Immun 1971, 3:694-701.

23. Ruiz M, Ewig S, Marcos MA, Martinez JA, Arancibia F, Mensa J, Torres $A$ : Etiology of community-acquired pneumonia: impact of age, comorbidity, and severity. Am J Respir Crit Care Med 1999, 160:397-405

24. Lieberman D, Schlaeffer F, Lieberman D, Horowitz S, Horovitz O, Porath A: Mycoplasma pneumoniae community-acquired pneumonia: a review of I0I hospitalized adult patients. Respiration 1996, 63:261-266.

25. Kauppinen M, Saikku P: Pneumonia due to Chlamydia pneumoniae: prevalence, clinical features, diagnosis, and treatment. Clin Infect Dis 1995, 2 I (suppl 3):S244-252.

26. Feldman C, Kallenbach JM, Levy H, Reinach SG, Hurwitz MD, Thorburn JR, Koornhof $\mathrm{HJ}$ : Community-acquired pneumonia of diverse etiology: prognostic features in patients admitted to an intensive-care unit and a "severity of illness"score. Intensive Care Med 1989, 15:302-307.

27. Valenti WM, Trudell RG, Bentley DW: Factors predisposing to oropharyngeal colonization with gram-negative bacilli in the aged. NEngl J Med 1978, 298: II08-IIIII.

28. Soler N, Torres A, Ewig S, Gonzalez J, Celis R, El-Ebiary M, Hernandez $C$, Rodriguez-Roisin R: Bronchial microbial patterns in severe exacerbations of chronic obstructive pulmonary diseases (COPD) requiring mechanical ventilation. Am J Respir Crit Care Med 1998, 157:1498-1505.

29. Song JH, Lee NY, Ichiyama S, Yoshida R, Hirakata Y, Fu W, Chongthaleong A, Aswapokee N, Chiu CH, Lalitha MK, Thomas K, Perera J, Yee TT, Jamal F, Warsa UC, Vinh BX, Jacobs MR, Appelbaum PC, Pai $\mathrm{CH}$ : Spread of drug-resistant Streptococcus pneumoniae in Asian countries: Asian network for surveillance of resistant pathogens (ANSORP) study. Clin Infect Dis 1999, 28: I206-121I.

30. Wang H, Hubener R, Chen MJ, Klugman K: Antibiotic susceptibility patterns of Streptococcus pneumoniae in China and comparison of MICs by agar dilution and E-Test methods. Antimicrob Agents Chemother 1998, 42:2633-2636.

31. Jiatai L, Weinstein AJ, Min Y, on behalf of China Bacterial Resistance Surveillance Study Group: Surveillance of bacterial resistance in China (1998-1999). Natl Med J China 200I, 81:8-I5.

32. Sahm DF, Jones ME, Hickey M, Diakun DR, Mani SV, Thornsberry C: Resistance surveillance of Streptococcus pneumoniae, Haemophilus influenzae and Moraxella catarrhalis isolated in Asia and Europe, 1997-1 998. J Antimicrob Chemother 2000, 45:457-466.

33. Ho PL, Yung RW, Tsang DN, Que TL, Ho M, Seto WH, Ng TK, Yam WC, Ng WW: Increasing resistance of Streptococcus pneumoniae to fluoroquinolones: results of a Hong Kong multicentre study in 2000. Antimicrob Chemother 200I, 48:659-665.

34. Niederman MS, Mandell LA, Anzueto A, Bass JB, Broughton WA, Campbell GD, Dean N, File T, Fine MJ, Gross PA, Martinez F, Marrie T], Plouffe JF, Ramirez J, Sarosi GA, Torres A, Wilson R, Yu VL, American Thoracic Society: Guidelines for the management of adults with community-acquired pneumonia. Diagnosis, assess- 
ment of severity, antimicrobial therapy, and prevention. Am J Respir Crit Care Med 200I, 163:1730-I754.

35. Mills GD, Oehley MR, Arrol B: Effectiveness of $\beta$ lactam antibiotics compared with antibiotics active against atypical pathogens in non-severe community acquired pneumkonia: meta-analysis. BMJ 2005, 330:456.

36. Gleason PP, Meehan TP, Fine JM, Galusha DH, Fine MJ: Associations between antimicrobial therapy and medical outcomes for hospitalized elderly patients with pneumonia. Arch Intern Med 1999, 159:2562-2572.

37. Garcia Vazquez E, Mensa J, Martinez JA, Marcos MA, Puig J, Ortega M, Torres A: Lower mortality among patients with communityacquired pneumonia treated with a macrolide plus a betalactam agent versus a beta-lactam agent alone. Eur J Clin Microbiol Infect Dis 2005, 24: 190-195.

38. Pechere JC, Gootz TD: Bacteriological activity of trovafloxacin, a new quinolone, against respiratory tract pathogens. Eur J Clin Microbiol Infect Dis 1998, 17:405-4I2.

39. MacGowan AP: Moxifloxacin (Bay 12-8039): a new methoxy quinolone antibacterial. Expert Opin Investig Drugs 1999, 8:181-199.

\section{Pre-publication history}

The pre-publication history for this paper can be accessed here:

http://www.biomedcentral.com/1471-2334/9/31/prepub

Publish with Bio Med Central and every scientist can read your work free of charge

"BioMed Central will be the most significant development for disseminating the results of biomedical research in our lifetime. "

Sir Paul Nurse, Cancer Research UK

Your research papers will be:

- available free of charge to the entire biomedical community

- peer reviewed and published immediately upon acceptance

- cited in PubMed and archived on PubMed Central

- yours - you keep the copyright

Submit your manuscript here:

http://www.biomedcentral.com/info/publishing_adv.asp 